\title{
Biocontrol and PGP potential of endophytic actinobacteria from selected ethnomedicinal plants in manipur, India
}

\begin{abstract}
The emergence of drug resistance in many bacterial pathogens and the rise of fungal infections have caused a resurgence of interest in finding new reserves of bioactive compounds. Manipur, a part of Indo-Burma hotspot, is rich in ethnomedicinal plants with folk medicinal and cultural significance. We have isolated 104 endophytic actinobacteria from 8 ethnomedicinal plants of Manipur. Eighty one (81) putative endophytic actinomycetes were selected for primary screening by cross streak method against bacterial test organisms (Micrococcus luteus, Bacillus subtilis, Escherichia coli and Pseudomonas aeruginosa). Thirty seven (37) isolates showed antibacterial activity and were further screened for other antimicrobial assays (Kirby Bauer and Dual Culture methods) against the same bacterial test organisms as well as indicator rice fungal pathogens (Curvularia oryzae, Rhizoctonia solani, Aspergillus niger, Bipolaris oryzae, Fusarium oxysporum and Pyricularia oryzae). In addition, they were subjected to various biochemical and physiological characterization tests and were also screened for plant growth promoting traits such as production of IAA, siderophore, and ammonia and solubilization of phosphate. Of 21 antagonistic isolates, 2 (CcRz4 and CaR1) were found most potent and they hold promise for further development as biocontrol agents. Optimization studies of these bioactive strains could be targets for future studies leading to biotechnological exploitation of these candidate endophytic bacteria.
\end{abstract}

Keywords: endophytic actinomycetes, antimicrobial assays, characterization tests, plant growth promoting (PGP) activities, biocontrol, manipur
Volume 4 Issue 6 - 2017

\author{
Khullakpam Shaheen, Saikat Mukherjee, \\ Debananda S Ningthoujam \\ Department of Biochemistry, Manipur University, India
}

Correspondence: Debananda S Ningthoujam, Department of Biochemistry, Microbial Biotechnology Research Laboratory (MBRL), Manipur University, Canchipur, Imphal 795 003, India, Email debananda.ningthoujam@gmail.com

Received: January 29, 2017 | Published: May 22, 2017

\section{Introduction}

Actinomycetes are unparalleled sources of bioactive natural products. ${ }^{1}$ Among microbes producing bioactive secondary metabolites, actinomycetes are the most prolific. ${ }^{1-5}$ Hence, survey of novel habitats and biotopes are warranted for discovery of novel actinomycetes and new secondary metabolites. Medicinal plants are a promising resource for isolation and screening of novel bioactive actinobacteria.

There has been a significant decline in the identification of new compounds due to re-discovery of known compounds from previously described terrestrial microbial species. ${ }^{6}$ Hence, investigation of novel and unique ecosystems for isolation of actinomycetes and for natural product-based drug discovery is crucial to overcome the problem of antibiotic resistance associated with the existing antibiotics. This trend has spurred interest in exploring the actinomycete diversity in marine environments. Not only are oceans a resource for biodiversity because of the physical properties of different ecosystems but also because many marine organisms have established endo-symbiotic and host-associated relationships with microorganisms. ${ }^{7,8}$

One biologically important but relatively overlooked niche is the inner tissues of higher plants. Endophytic actinobacteria are relatively unexplored for potential sources of novel species and novel natural products for biomedical, agricultural and industrial exploitation. The last few years has seen acceleration in research on endophytic microbes inhabiting the plants from various ecosystems. ${ }^{9-16}$

Endophytic actinobacteria have attracted attention in recent years with increasing reports of isolates from a range of plant types including crop plants and medicinal plants. They have been demonstrated to improve and promote the growth of host plants as well as to reduce disease symptoms caused by plant pathogens through various mechanisms, including production of secondary metabolites, changes in host physiology, and induction of systemic acquired resistance in plants. ${ }^{17}$ Endophytes provide a wide variety of bioactive secondary metabolites with unique structures including alkaloids, benzopyranones, chinones, flavonoids, phenolic acids, quinones, steroids, terpenoids, tetralones, xanthones, and other classes. ${ }^{18}$ Such bioactive metabolites find wide-ranging applications as agrochemicals, antibiotics, immunosuppressants, antiparasitics, antioxidants, and anticancer agents. ${ }^{19}$ Bioactive natural compounds produced by endophytes have shown greater promise over synthetic chemicals from safety and human health perspectives, although there is still a significant global demand for synthetic products due to economic and time-saving reasons. ${ }^{20}$

Continuous use of fungicides in agriculture has resulted in increased fungicide resistance, environmental pollution, detrimental effects on non-target organisms including human beings. ${ }^{21}$ In recent years, natural or biological control has received increased attraction by providing an environment friendly, long lasting, inexpensive, and safe alternative for control of plant diseases..$^{22,23}$ Increased efforts have been made to screen micro-organisms for bioactive molecules antagonistic to plant pathogens. There are many reports on the use of microorganisms as biocontrol agents as an alternative to chemical fungicides. ${ }^{24,25}$ Among micro-organisms, actinomycetes of the genus Streptomyces are well known for their antagonistic potential against a wide range of fungal phytopathogens,.$^{26}$ Many Streptomyces spp. have been reported to produce fungal cell wall degrading enzymes 
such as chitinases, hemicellulases, cellulases and glucanases ${ }^{27,28}$ and the role of these enzymes in antifungal activity and biocontrol potential has been evaluated. ${ }^{29}$ They are also known for their ability to promote plant growth by producing plant growth hormones such as indole acetic acid, nitrogen fixation, phosphate solubilization and siderophore production. . $^{30,31}$

Manipur lies in the Indo-Burma hotspot in India, one of the 34 sites in the world ${ }^{32}$ that abuts the South East Asian tropical Rainforests. Though small, it has a variety of ecological zones, niche habitats and other unique biotopes. There is a great potential for discovery of bioactive actinomycete strains in Manipur for beneficial applications in medicine, agriculture and industry. There is an urgent need to explore such underexplored ecosystems of Manipur and study the distribution of actinomycetes by isolating, characterizing and screening them for discovery of novel actinomycetes and development of novel natural products with commercial biotechnological applications. In the present study, we selected some medicinal plants of Manipur for possible isolation of novel actinomycetes. The aim of the investigation was to isolate and characterize putative actinomycete and other bacterial strains, and screen their biocontrol (antagonistic) and plant growth promotion (PGP) activities.

\section{Material and methods}

\section{Sampling of ethnomedicinal plants}

Healthy roots, leaves, rhizomes, and stems of each plant were collected and placed in sterile bags and taken to the laboratory. The samples were subjected to isolation procedures within $96 \mathrm{~h}$.

8 ethnomedicinally important plants of Manipur were sampled from various parts of the state:

\section{Scutellaria discolor (local name- Yetnakhat)}

II. Curcuma caesia(local name- Yai-mu)

III. Paris polyphylla (local name- Sing-pan)

IV. Centella asiatica (local name- Peruk)

V. Alium odorum (local name- Maroi-nakuppi)

VI. Catharanthes roseus(local name- Saheb- lei)

VII. Achryranthes aspera(local name- Khujum- pere)

VIII. Kaempferia rotunda(local name-Yai Thamna Manbi).

\section{Isolation}

Samples were washed with Tween 20, soaked in distilled water, and vortexed to remove the surface soils. After drying, the samples were subjected to a five step surface sterilization procedure. ${ }^{33}$

After thorough washing, the samples were air dried under sterile conditions. The surface treated samples were cut into small pieces $(1 \mathrm{~cm})$ with a sterile blade and inoculated into Starch Casein Nitrate Agar (SCNA), 2.5\% Water Agar (WA), Tap Water Yeast Extract Agar (TWYE) and Tap Water Peptone Agar (TWPA). The inoculated plates were then incubated at $30^{\circ} \mathrm{C}$ for $3-4$ weeks. Morphologically distinct isolates were subcultured till pure cultures were obtained.

\section{Bioactivity screening}

Primary screening (Cross streak method): The endophytic isolates were cross-streaked ${ }^{34,35}$ against indicator test organisms viz.
Micrococcus luteus (MTCC-106), Bacillus subtilis (MTCC-121), Escherichia coli (MTCC-739), Pseudomonas aeruginosa (DN1). The isolates were streaked in the middle of the Nutrient Agar plates and kept for 3-4days at $30^{\circ} \mathrm{C}$. Freshly grown test organisms (MTCC-106, MTCC-121, MTCC-739, DN1) were streaked perpendicularly to the actinomycete isolate, incubated for $24-48 \mathrm{~h}$ and the zones of inhibition were measured. The isolates which showed inhibition of $>50 \%$ against the test organisms in the primary screening were considered to be potent.

Secondary screening (Kirby Bauer method): The bioactive strains were subjected to secondary screening for antibacterial activity against the same bacterial test organisms (MTCC-106, MTCC-121, MTCC-739 and DN1) using Kirby-Bauer method. ${ }^{36}$

The strain was inoculated on Starch Casein Nitrate (SCN) Broth and kept at $30^{\circ} \mathrm{C}$ on an orbital shaker (150rpm, 7days). The fully grown putative actinomycete cultures were centrifuged $(10,000 \mathrm{rpm}$, $15 \mathrm{~min}$ ) and the supernatants were collected in sterile falcon tubes. The test organisms were inoculated in test tubes containing Nutrient Broth (NB) and kept at $30^{\circ} \mathrm{C}$ for $24 \mathrm{~h} .100 \mu \mathrm{l}$ of freshly grown test organism was spread plated on Nutrient Agar (NA, pH 7.0) and kept for drying. Agar wells $(6 \mathrm{~mm}$ diameter) were punched into the dried plates and filled with $0.7 \%$ soft agar up to $1 / 2$ depths of the wells. The culture supernatants were then put into the wells in duplicates and kept at $4^{\circ} \mathrm{C}$ for $3 \mathrm{~h}$ for proper diffusion. The plates were incubated at $30^{\circ} \mathrm{C}$ for $4-5$ days and then observed for the presence or absence of inhibition zones around the wells.

Biocontrol assays against fungal pathogens (Dual culture method): The bioactive strains were screened for antifungal activities against major rice fungal pathogens viz. Curvularia oryzae (MTCC 2605), Rhizoctonia solani (MTCC 4633), Aspergillus niger (MTCC 1344), Bipolaris oryzae (LSMU1), Fusarium oxysporum (MTCC 287) and Pyricularia oryzae (MTCC 1477) using the Dual culture method. ${ }^{37}$

An in vitro plate technique was used to monitor the inhibitory (antagonistic) effects of the actinomycetes against the fungal pathogens. This test was done on SDA (Sabouraud Dextrose Agar) plates. Agar wells (6mm diameter) were punched on SDA plates using sterilized cork borer and agar plugs from the freshly grown cultures were placed on the SDA wells in duplicates. The plates were incubated at $30^{\circ} \mathrm{C}$ for $48 \mathrm{~h}$. For each test fungus, a $6 \mathrm{~mm}$ diameter SDA plug covered fully with the actively growing mycelium was placed at the centre of the plate. A plate containing a fungal agar plug without the culture was kept as control. All the plates were incubated at $30^{\circ} \mathrm{C}$ until the control plate showed full growth.

The percentage growth inhibition was calculated using the formula:

Inhibition $=[(\mathrm{R}-\mathrm{r}) / \mathrm{R}] \mathrm{X} 100$,

Where $\mathrm{R}=$ Radius of the fungal growth in control plate

$\mathrm{r}=$ Radius of the fungal growth in test plate.

\section{Phenotypic characterization}

All the bioactive isolates shortlisted after screening were subjected to various biochemical tests (starch hydrolysis, catalase, oxidase, MR, VP, citrate utilization, indole production, nitrate reduction, gelatin liquefaction and urease tests) and physiological characterization tests (effect of different salt concentrations and $\mathrm{pH}$ on growth) using standard procedures. ${ }^{38-41}$ 


\section{In vitro tests for plant growth promoting (PGP) traits}

The bioactive isolates were further screened for plant growth promoting activities such as production of IAA, siderophore and ammonia and Phosphate solubilization.

\section{Indole acetic acid (IAA) production}

IAA production was determined according to standard procedure. ${ }^{42}$ Strains were inoculated in Starch Casein Nitrate broth (SCNB) containing $2 \mathrm{mg} / \mathrm{ml}$ of L-tryptophan (trp) and incubated on an orbital shaker $\left(150 \mathrm{rpm}, 30^{\circ} \mathrm{C}, 5 \mathrm{~d}\right)$. The culture broths were centrifuged $(10,000 \mathrm{rpm}, 10 \mathrm{~min})$ and $1 \mathrm{ml}$ of the supernatant was mixed with $2 \mathrm{ml}$ of Salkowski reagent. Appearance of pink color indicated IAA production.

\section{Siderophore production}

Siderophore production was assayed according to standard protocol ${ }^{43}$ with some modifications. Agar plugs $(6 \mathrm{~mm}$ diameter) containing strains were inoculated on SCNA (without iron) amended with CAS-substrate and kept incubated at $30^{\circ} \mathrm{C}$ for $7 \mathrm{~d}$. Orange coloured halo zones surrounding the colonies were indicative of siderophore production.

\section{Phosphate (P) solubilization}

Solubilization of tricalcium phosphate (TCP) was determined by spot inoculation of a loopful of the isolate in National Botanical
Research Institute's Phosphate Growth Medium containing bromophenol blue (NBRIP-BPB) ${ }^{44}$ The strain fully grown on SCNA was spot inoculated in NBRIP-BP medium and kept incubated at $30^{\circ} \mathrm{C}$. Formation of a halo zone surrounding the colony after $4 \mathrm{~d}$ indicated P solubilization.

\section{Ammonia production}

Ammonia production was screened in peptone water. Strains were inoculated in $10 \mathrm{ml}$ peptone water (broth) and kept in a shaker $\left(150 \mathrm{rpm}, 30^{\circ} \mathrm{C}\right)$ for $4 \mathrm{~d} .0 .5 \mathrm{ml}$ of Nessler's reagent was then added in each tube. Development of brown to yellow color indicated ammonia production. ${ }^{38}$

\section{Results}

\section{Sampling of medicinal plants \& isolation of endophytic actinomycetes}

Eight ethnomedicinally important plants of Manipur were sampled from various parts of the state (Figure 1). Putative actinomycete colonies on master plates (Figure 2) were subcultured till pure cultures were obtained. A total of 104 endophytic actinomycete isolates were obtained.

\section{Bioactivity Screening of endophytic isolates}

81 putative endophytic actinomycetes were selected for antimicrobial assays.

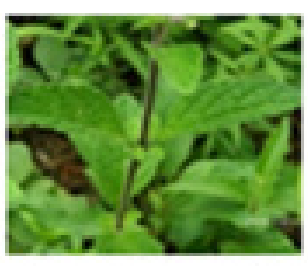

Sencellteria dischler

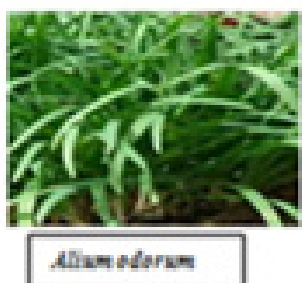

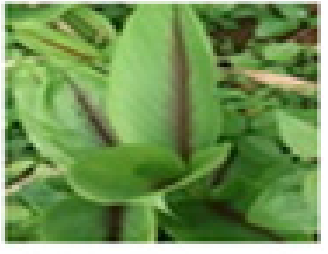

Curcume estala

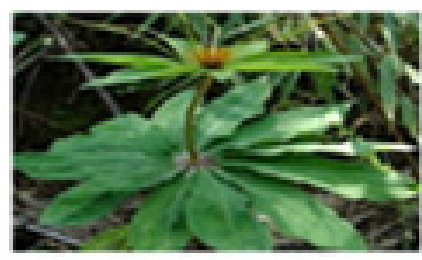

Peria poloble

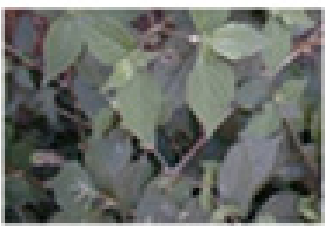

Ackprocken apera

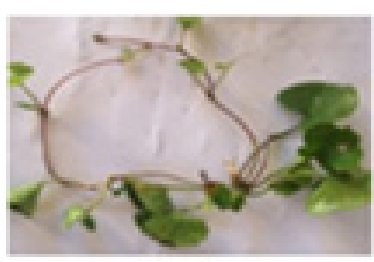

Cererillt asiesiet

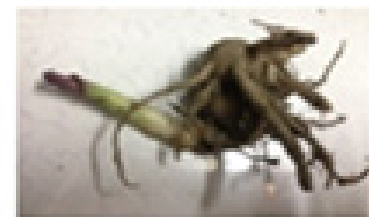

Eernferia reande

Catkertecken noses

Figure I Plant samples used for endophytic actinomycete isolation:The figure represents the 8 ethnomedicinally important plants of Manipur sampled during the study for endophytic actinomycete isolation namely Scutellaria discolour, Curcuma caesia, Paris polyphylla, Centella asiatica, Alium odorum, Catharanthes roseus, Achryranthes aspera and Kaempferia rotunda (with local names Yetnakhat, Yai-mu, Sing-pan, Peruk, Maroi-nakuppi, Saheb- lei, Khujum- pere, and Yai Thamna Manbi respectively).
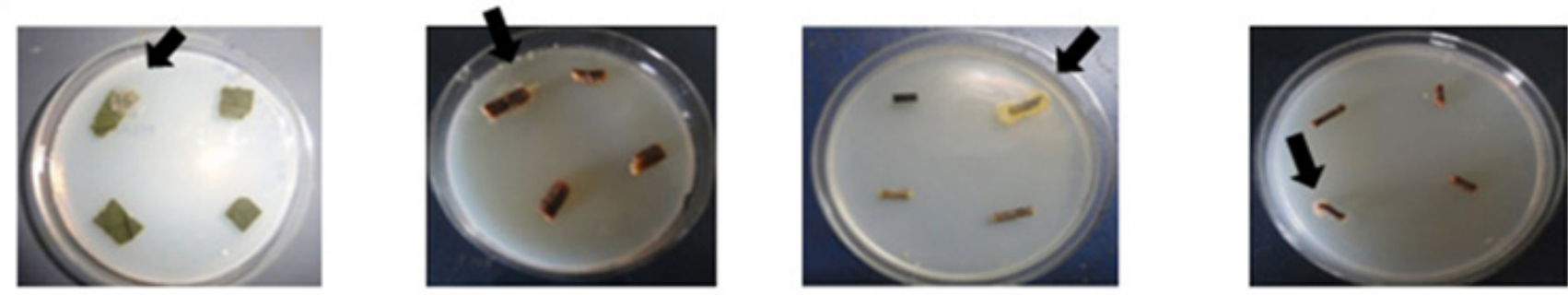

Figure 2 Master plates of endophytic actinomycetes: The figure represents the master plates of endophytic actinomycetes isolation showing the bacterial colonies (indicated by arrows) which were sub-cultured till pure cultures were obtained.A total of I04 endophytic actinomycete isolates were obtained. 


\section{Primary screening (Cross streak method)}

Of 81 putative endophytic actinomycete isolates screened, 37 showed antibacterial activity against one or more of the test organisms (Table 1) of which 9 strains (SdL4, CcRz1, CcRz3, CcRz7, CcRz9, CcRz18, CcRz22, CaL6, CaR1) were found to be potent.

Table I Primary screening of bioactive endophytic actinomycete strains

\begin{tabular}{|c|c|c|c|c|c|}
\hline \multirow{2}{*}{ SI. no } & \multirow{2}{*}{ Actinomycete strains } & \multicolumn{4}{|c|}{ Test organisms } \\
\hline & & MTCC 106 & MTCC I2I & MTCC 739 & DNI \\
\hline I & SdL4 & +++ & ++ & - & - \\
\hline 2 & CcRzl & ++ & + & - & - \\
\hline 3 & CcRz3 & - & ++ & - & - \\
\hline 4 & CcRz7 & - & ++ & - & - \\
\hline 5 & CcRz9 & - & +++ & - & - \\
\hline 6 & CcRzl8 & - & ++ & - & - \\
\hline 7 & $\mathrm{CcRz} 22$ & - & +++ & ++ & - \\
\hline 8 & CaL6 & +++ & + & - & - \\
\hline 9 & CaRI & ++ & ++ & + & + \\
\hline
\end{tabular}

Note: $+++(>50 \%),++(<50 \%),+(<30 \%),-$ no inhibition zone

\section{Secondary screening (Kirby bauer method)}

All 37 bioactive actinomycete strains were subjected to secondary screening for antibacterial activity against the same bacterial test organisms. Eleven (11) endophytic strains showed antimicrobial activity against one or more of the test organisms of which 4 strains (CcRz22, CaL6, CaR5, AoS2) were found to be promising. None of the isolates showed any activity against Pseudomonas aeruginosa (Table 2 \& Figure 3).

Table 2 Profiling of antibacterial activity of bioactive actinomycete strains (Kirby Bauer method)

\begin{tabular}{|c|c|c|c|c|c|}
\hline \multirow{2}{*}{ SI. no } & \multirow{2}{*}{ Actinomycete strains } & MTCC I06 & MTCC I2I & MTCC 739 & DNI \\
\hline & & \multicolumn{4}{|c|}{ Inhibition zone (mm) } \\
\hline I & SdL4 & 13 & 11.6 & - & - \\
\hline 2 & SdL5 & - & - & - & - \\
\hline 3 & CcRzl & 14.2 & 10.4 & - & - \\
\hline 4 & CcRz7 & - & 15 & - & - \\
\hline 5 & CcRz9 & - & 16.7 & - & - \\
\hline 6 & CcRzI8 & - & 12 & - & - \\
\hline 7 & CcRz22 & - & 27.3 & 11.1 & - \\
\hline 8 & CaRI & 15.6 & 12.1 & 15.2 & - \\
\hline 9 & CaR4 & 13.9 & 15.9 & 16.1 & - \\
\hline 10 & CaR5 & 19.4 & 17.6 & 18.6 & - \\
\hline II & AoS2 & 13.6 & 24.2 & - & - \\
\hline
\end{tabular}

An inhibition zone of $17 \mathrm{~mm}$ or above is considered potent.

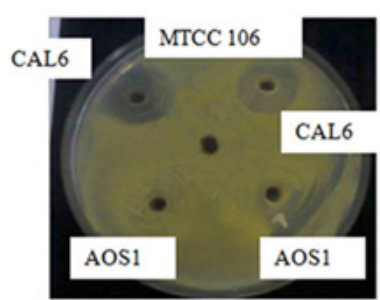

Figure 3 Antimicrobial assay by Kirby Bauer method: The figure represents the plates of antimicrobial assay by Kirby Bauer method of the strains CaL6 and AoSI showing antibacterial activity against the test organism MTCC I06 (Micrococcus luteus) while AoS2 and CcRz22 against MTCC-I2I (Bacillus subtilis ) .

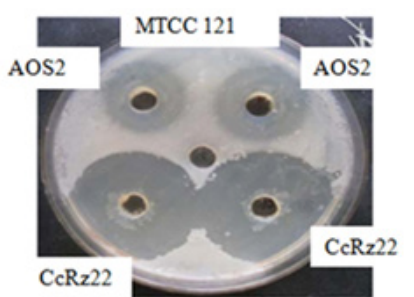

Biocontrol assays against fungal pathogens (Dual culture method)

All the 37 bioactive endophytic isolates were screened for antifungal activities against indicator rice fungal pathogens. Twenty one (21) isolates were found to have antifungal activity against one or more of the test organisms, of which 2 strains (CcRz4 and CaR1) were found to be most potent (Table $3 \&$ Figure 4).

\section{Phenotypic characterization}

Biochemical tests: Majority of the bioactive strains were found positive in starch hydrolysis and negative in indole production tests. 21 strains showed positive results in starch hydrolysis, 11 in catalase, 
12 in oxidase, 9 in MR, 6 in VP, 20 in citrate utilization, 29 in nitrate reduction, 10 in gelatin liquefaction and 11 in urease tests. Only 1 strain (CcRz24) was positive in indole production test.

Effect of salt concentration: None of the bioactive strains except CcRz6 could grow at $10 \% \mathrm{NaCl}$ concentration. CcRz6 could grow at $0-10 \% \mathrm{NaCl}$ concentrations. One strain (CcRz12) could grow at $0.5-10 \% \mathrm{NaCl}$ concentrations. CaR1 grew luxuriantly at lower $\mathrm{NaCl}$ concentrations $(0-1 \%)$ but was unable to grow at higher salt concentrations. One strain, PpRz3, grew luxuriantly at $0-2.5 \% \mathrm{NaCl}$ concentrations but failed to grow at higher salt concentrations). The remaining strains grew luxuriantly at $0-7 \% \mathrm{NaCl}$ concentrations.

Table 3 Percentage growth inhibition of the fungal pathogens by the bioactive strains

\begin{tabular}{|c|c|c|c|c|c|c|c|}
\hline SI. no & Test isolates & \% Mycelial Gr & the $=[(R-r) / R]$ & & & & \\
\hline \multirow{2}{*}{ Control } & & MTCC 4633 & MTCC 287 & MTCC 2605 & MTCC I344 & LSMUI & MTCC 1477 \\
\hline & & 0 & 0 & 0 & 0 & 0 & 0 \\
\hline I & SdL2 & 72.5 & 66 & 65.9 & - & - & - \\
\hline 2 & SdL3 & - & - & - & - & - & $64.286 \pm 1.414$ \\
\hline 3 & SdL5 & 62.9 & - & 62.1 & - & - & - \\
\hline 4 & $\mathrm{CcRz} 3$ & - & - & 64.4 & - & - & $61.904 \pm 1.414$ \\
\hline 5 & CcRz4 & 69.8 & 70.1 & 72.9 & 68.2 & 59.9 & $65.676 \pm 2.121$ \\
\hline 6 & CcRzI2 & - & - & - & - & - & $61.904 \pm 4.242$ \\
\hline 7 & CcRzI3 & - & - & - & - & - & $64.286 \pm 4.242$ \\
\hline 8 & CcRzl7 & - & - & - & - & - & $53.57 I \pm 3.535$ \\
\hline 9 & CcRz2I & 30.2 & - & - & - & - & $55.953 \pm 0.707$ \\
\hline 10 & CcRz22 & 32.6 & - & - & - & - & $58.333 \pm 0.707$ \\
\hline II & PpRzI & - & - & - & - & - & $66.667 \pm 1.414$ \\
\hline 12 & $\mathrm{PpRz2}$ & - & - & - & - & - & $61.904 \pm 1.414$ \\
\hline 13 & PpRz4 & - & - & - & - & - & $61.904 \pm 0.000$ \\
\hline 14 & CaL2 & - & - & - & - & - & $58.333 \pm 4.949$ \\
\hline 15 & CaL3 & - & - & - & - & - & $65.476 \pm 0.707$ \\
\hline 16 & CaL5 & - & - & 33.6 & - & - & $61.904 \pm 1.414$ \\
\hline 17 & CaL6 & 59.6 & 66.9 & 48.1 & - & - & $59.524 \pm 2.828$ \\
\hline 18 & CaS4 & - & - & - & 76.19 & 64.04 & - \\
\hline 19 & CaRI & 33.4 & 75.9 & 65.9 & 64.8 & 75.9 & $57.143 \pm 1.414$ \\
\hline 20 & AoS2 & - & - & - & - & - & $57.143 \pm 0.000$ \\
\hline 21 & VrL2 & - & - & - & - & - & $69.048 \pm 0.000$ \\
\hline
\end{tabular}
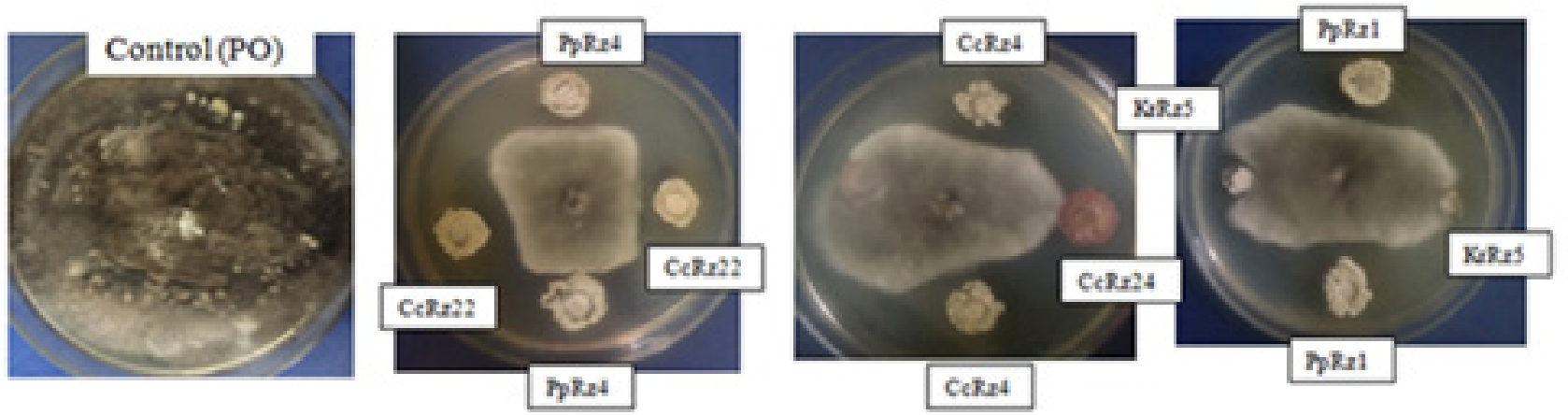

Figure 4 Biocontrol assay by dual culture method: The figure represents the plates of biocontrol assay by dual culture method of the strains PpRz4, CcRz22, CcRz24, CcRz4, KrRz5 and PpRzI showing antifungal activity against the rice fungal pathogen Pyricularia oryzae/ MTCC I477 (PO).

Effect of pH on growth: All the strains grew luxuriantly at pH 7-9. pH 7-10. 11 strains (CcRz8, CcRz12, CcRz13, PpRz1, PpRz2, PpRz3, 7 strains (SdL2, SdL3, CcRz3, CcRz4, CcRz18, AoS2 and KrRz5) showed no growth at $\mathrm{pH} 5$ and 6 , though they could grow at $\mathrm{pH} 7-9$ or

PpRz4, CaL2, CaL6, CaR1 and VrL2) failed to grow at $\mathrm{pH} 5$ but grew well at $\mathrm{pH}$ 6-10. The rest of the bioactive isolates grew at $\mathrm{pH}$ 5-10. 


\section{In vitro tests for plant growth promoting (PGP) traits}

Twenty-eight (28) strains showed positive results for IAA production while 14 were positive for siderophore production, 20 for phosphate solubilization test and 27 strains for ammonia production (Table 4, Figures $5 \& 6$ ).

Table 4 Screening for plant growth promoting (PGP) activities

\begin{tabular}{|c|c|c|c|c|c|}
\hline SI.no. & Strains & IAA production & Siderophore production & Phosphate solubilization & Ammonia production \\
\hline 1 & CcRzl & + & + & + & + \\
\hline 2 & CcRz3 & + & + & - & + \\
\hline 3 & CcRz4 & + & - & + & + \\
\hline 4 & CcRz6 & + & + & + & + \\
\hline 5 & CcRz7 & + & - & + & + \\
\hline 6 & CcRz9 & + & + & + & + \\
\hline 7 & CcRzI2 & + & - & + & + \\
\hline 8 & CcRzI7 & + & - & + & + \\
\hline 9 & CcRz2I & + & + & + & + \\
\hline 10 & CcRz24 & + & - & + & + \\
\hline 11 & PpRzl & + & + & - & + \\
\hline 12 & PpRz4 & + & - & + & + \\
\hline 13 & CaL3 & + & - & + & + \\
\hline 14 & CaL6 & + & - & + & + \\
\hline 15 & CaS4 & + & + & + & + \\
\hline 16 & AoSI & + & + & + & + \\
\hline 17 & Aos2 & + & + & - & + \\
\hline 18 & VrL2 & + & + & - & + \\
\hline 19 & VrS4 & + & + & + & + \\
\hline 20 & KrRz5 & + & + & - & + \\
\hline
\end{tabular}
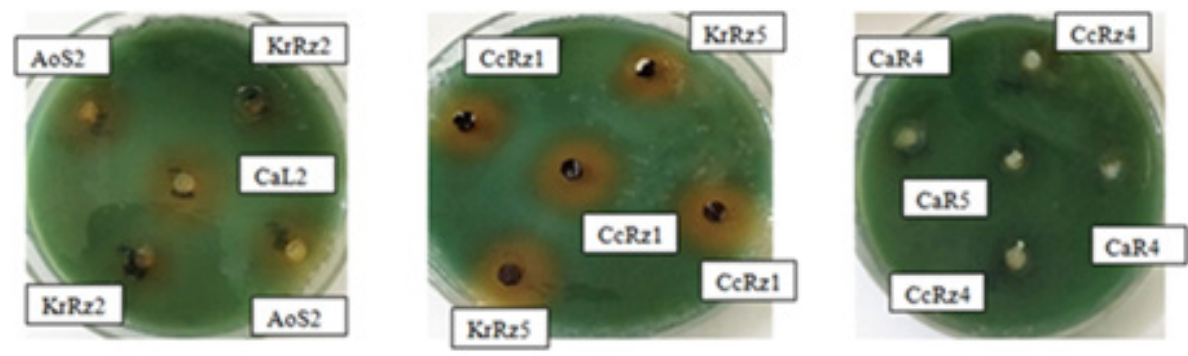

Figure 5 In vitro tests for siderophore production: The figure represents the plates of in vitro tests for siderophore production of the four strains (AoS2, CaL2, $\mathrm{CcRzI}$ and $\mathrm{KrRz} 5$ ) showing positive results and the four strains (KrRz2, $\mathrm{CaR4}, \mathrm{CaR5}$ and $\mathrm{CcRz} 4)$ showing negative results.
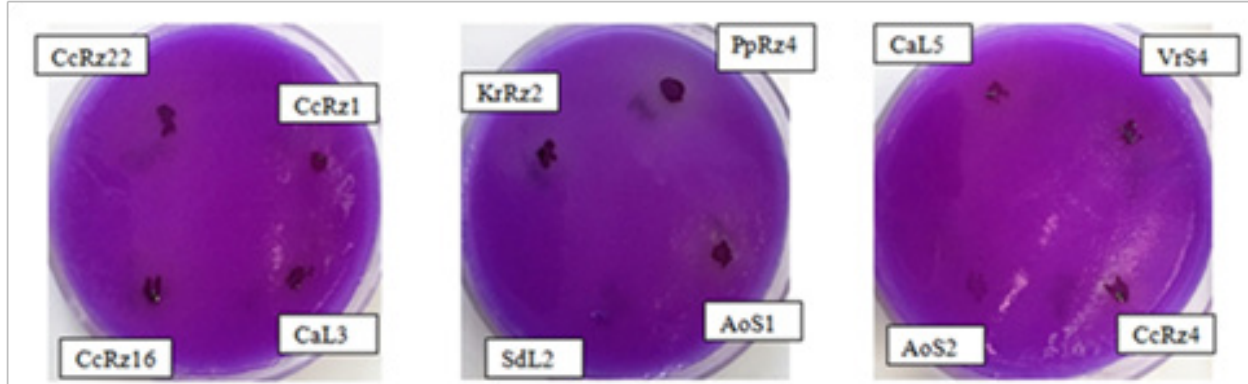

Figure 6 In vitro tests for phosphate solubilisation: The figure represents the plates of in vitro tests for phosphate solubilisation of the ten strains (CcRz22, CcRzI6, CaL3, CcRzI, AoSI, KrRz2, PpRz4, CaL5,VrS4 and CcRz4) showing positive results and the two strains (AoS2 and SdL2) showing negative results. 


\section{Discussion}

Plants growing in great areas with rich biodiversity are likely to house endophytes with equal or greater biodiversity. Plants with an ethnobotanical history and unusual longevity are expected to be more potent sources of endophytes producing active natural products. ${ }^{20}$ In this study, we have isolated 104 putative endophytic actinomycetes from eight ethnomedicinally important plants of Manipur. Eightyone (81) putative endophytic strains were selected for antimicrobial assays. Zhao et al., ${ }^{45}$ reported 560 endophytes from 26 species of healthy medicinal plants where more number of isolates were recovered from roots $(58.2 \%)$, followed by stems $(27.8 \%)$ and leaves $(14 \%) .{ }^{45}$ Actinomycetes have been indicated as a large part of the endophytic microbial flora. ${ }^{46}$

As described in earlier studies, the strains we isolated possessed more antifungal than antibacterial properties..$^{47,48}$ Primary screening revealed that 37 of 81 isolates have antibacterial activity against one or more of the test organisms of which 9 were potent. In Secondary screening, 11 isolates exhibited antimicrobial activity, of which 4 were found to be potent. None of the isolates were found to have any activity against Pseudomonas aeruginosa. Biocontrol assay against six rice fungal pathogens indicated that 21 isolates have antifungal activity against one or more of the test organisms, of which CcRz4 and CaR1 were found to be the most potent and they hold promise for further development as biocontrol agents. The results of this study indicate that these endophytic isolates from medicinal plants in Manipur have potential to be used as biological control agents against fungal phytopathogens. Preliminary characterization of these 37 bioactive actinomycete strains by biochemical and physiological tests gave results typical of actinomycetes. ${ }^{49}$

There are many reports which demonstrated the ability of endophytic and rhizospheric soil Streptomyces to produce indole acetic acid and thus promote plant growth. ${ }^{50,51}$ Twenty-eight of our isolates produced IAA. Endophytic actinomycetes were reported to produce IAA ranging between $9.85-15.4 \mu \mathrm{g} / \mathrm{ml} .{ }^{52}$ In agriculture, biological phosphate solubilization as an alternative to natural phosphate utilization plays an important role in efficient nutrient uptake. 20 endophytic isolates were positive for $\mathrm{P}$ solubilization and 27 for ammonia production. In addition, 14 isolates produced siderophore. Siderophore production may be involved in the inhibition of pathogens and, indirectly in promotion of plant growth. ${ }^{53-55}$

Our study revealed that medicinal plants in Manipur are a promising source of endophytic bacteria with biocontrol and PGP potential. Further studies are underway to assess the agricultural applications of these endophytic strains.

\section{Conclusion}

Among 37 endophytic actinomycetes screened for antimicrobial activities, 4 (CcRz22, CaL6, CaR5, AoS2) strains were found to be promising. The strain CcRz4 and CaR1 were found to be most promising biocontrol strains. Seven strains (CcRz1, CcRz6, CcRz9, CcRz21, CaS4, AoS1 and VrS4) showed positive results in all the PGP traits tested. Optimization studies of these bioactive strains by changing cultural conditions could be targets of future studies leading to biotechnological exploitation of these bioactive strains. The study also corroborate that Manipur (a part of Indo-Burma hotspot) holds great potential for discovery of novel actinomycetes and development of novel natural products with commercial biotechnological applications.

\section{Acknowledgements}

The authors would like to gratefully acknowledge the Department of Biotechnology (DBT), Govt. of India, for grant towards the SBT Hub scheme, Department of Biochemistry, Manipur University (BT/04/NE/2009) that partly facilitated this work.

SK thanks the Department of Science \& Technology (DST), Govt. of India for providing the INSPIRE Fellowship for PhD work (DST/ INSPIRE Fellowship/2014/195).

\section{Author's contributions}

Khullakpam Shaheen conducted the experiments and wrote the initial draft of the manuscript. Debananda S. Ningthoujam designed and supervised the study and gave critical, analyses, reviews and comments. Saikat Mukherjee helped edit the initial versions of the manuscript.

\section{Conflict of interest}

The author declares no conflict of interest.

\section{References}

1. Berdy J. Bioactive microbial metabolites. J Antibiotics. 2005;58(1):1-26.

2. Saadoun I, Al-Momani F. Activity of North Jordan soil streptomycete isolates against Candida albicans. World J Microbiol Biotechnol. 2000;16(2):139-142.

3. Zhao H, Kassama Y, Young M, et al. The rapid differentiation of Streptomyces isolates using Fourier transform infrared spectroscopy. Vibrational Spectroscropy. 2006;40(2):213-218.

4. Zin NM, Sarmin NI, Ghadin N, et al. Bioactive endophytic streptomycetes from the Malay Peninsula. FEMS Microbiol Lett. 2007;274(1):83-88.

5. Kekuda PTR. Purification, characterization and pharmacological activities of bioactive metabolites from potent actinomycetes strains. India: Kuvempu University; 2014.

6. Saravanakumar R, Moomeen HS, Ronald J, et al. Control of fish bacterial pathogens, by antagonistic marine actinomycetes isolated from Gulf of Mannar Coast. World J Fish \& Marine Sci. 2010;4:275-279.

7. Achtman M, Wagner M. Microbial diversity and the genetic nature of microbial species. Nat Rev Microbiol. 2008;6(6):431-440.

8. Abdelmohsen UR, Pimentel-Elardo SM, Hanora A, et al. Isolation, phylogenetic analysis and anti-infective activity screening of marine sponge- associated actinomycetes. Mar Drugs. 2010;8(3):399-412.

9. Ryan RP, Germaine K, Franks A, et al. Bacterial endophytes: recent developments and applications. FEMS Microbiol Lett. 2008;278(12):19 .

10. Zhang HW, Song YC, Tan RX. Biology and chemistry of endophytes. Nat Prod Rep. 2006;23(5):753-771.

11. Kafur A, Khan AB. Isolation of endophytic actinomycetes from Catharanthes roseus (L.) G. Don leaves and their antimicrobial activity. Iranian J Biotechnol. 2011;9(4):302-306.

12. Taechowisan $\mathrm{T}$, Chanaphat $\mathrm{S}$, Ruensamran $\mathrm{W}$, et al. Antibacterial activity of Decursin from Streptomyces sp. GMT-8; an endophyte in Zingiber officinale Rosc. J Appl Pharmaceutical Sci. 2013;3(10):74-78.

13. Selim KA, El-Beih AA, AbdEl-Rahman TM, et al. Biology of Endophytic Fungi. Curr Res Environ Mycol. 2012;2(1):31-82.

14. Golinska P, Wypij M, Agarkar G, et al. Endophytic actinobacteria of medicinal plants:diversity and bioactivity. Antonie van Leeuwenhoek. 2015;108(2):267-289. 
15. Passari AK, Mishra VK, Gupta VK, et al. In vitro and in vivo plant growth promoting activities and DNA fingerprinting of antagonistic endophytic actinomycetes associates with medicinal plants. PLoS One. 2015;10(9):e0139468.

16. Roy S, Banerjee D. Bioactive endophytic actinomycetes of Cinnamomum $s p$; Isolation, identification, activity guided purification and process optimization of active metabolite. American J Microbiol. 2015;6(1):4 13.

17. Qin S, Li J, Chen H-H, et al. Isolation, diversity, and antimicrobial activity of rare actinobacteria from medicinal plants of tropical rain forests in xishuangbanna, China. Appl Environ Microbiol. 2009;75(19):61766186.

18. Tan GYA, Mei YS. Actinobacterial Diversity in Limestone Caves of Peninsula Malaysia. Proceeding of the $15^{\text {th }}$ International Symposium on the Biology of Actinomycetes, Shanghai, China; 2009. 62 p.

19. Gunatilaka AAL. Natural products from plant-associated microorganisms: Distribution, structural diversity, bioactivity and implication of their occurence. J Nat Prod. 2006;69(3):509-526.

20. Strobel G, Daisy B, Castillo U. Natural products from endophytic microorganisms. J Nat Prod. 2004;67(2):257-268.

21. Cohen Y, Coffey MD. Systemic fungicides and the control of Oomycetes. Annu Rev Phytopathol. 1986;24:311-338.

22. Aghighi S, Shadidi Bonjar GH, Rawashdeh R, et al. First report of antifungal espectra of activity of Iranian actinomycetes strains against Alternaria solani, Alternaria alernata, Fusarium solani, Phytophthora megasperma, Verticillium dahliae, and Saccharomyces cerevisiae. Asian J Plant Sci. 2004;3(4):463-471.

23. Dhingra OD, Sinclair JB. Basic plant pathology methods. 1st ed. USA: CRC Press; 1995.

24. Welbaum G, Sturz AV, Dong Z, et al. Fertilizing soil microorganisms to improve productivity of agroecosystems. Crit Rev Plant Sci. 2004;23:175-197.

25. Singh AK, Chhatpar HS. Combined use of Streptomyces sp. A6 and chemical fungicides against Fusarium wilt of Cajanus cajan may reduce the dosage of fungicides used in the field. Crop Prot. 2011;30(7):770775 .

26. Gomes RC, Semedo LT, Soares RM, et al. Chitinolytic activity of Actinomycetes from a cerrado soil and their potential in biocontrol. Lett Appl Microbiol. 2000;30(2):146-150.

27. Petrosyan P, Gárcia-Varela M, Luz-Madrigal A, et al. Streptomyces mexicanus sp., a xylanolytic micro-organism isolated from soil. Int $J$ Syst Evol Microbiol. 2003;53(Pt 1):269-273.

28. Ramesh S, Mathivanan N. Screening of marine Actinomycetes isolated from the Bay of Bengal, India for antimicrobial activity and industrial enzymes. World J Microb Biotechnol. 2009;25(12):2103-2111.

29. Ding $\mathrm{CH}$, Jiang ZQ, Li XT, et al. High activity Xylanase production by Streptomyces olivaceoviridis E-86. World J Microbiol Biotechnol. 2004;20(1):7-10.

30. Cattelan ME, Hartel PG, Fuhrmann JJ. Screening of plant growthpromoting Rhizobacteria to promote early soybean growth. Soil Sci Soc Am. 1999;63:1670-1680.

31. Franco-Correa M, Quintana A, Duque C, et al. Evaluation of Actinomycete strains for key traits related with plant growth promotion and Mycorrhiza helping activities. Appl Soil Ecol. 2010;45:209-217.

32. http://www.conservation.org

33. Qin S, Li J, Chen $\mathrm{H}-\mathrm{H}$, et al. Isolation, diversity, and antimicrobial activity of rare actinobacteria from medicinal plants of tropical rain forests in xishuangbanna, China. Appl Environ Microbiol. 2009;75(19):61766186.
34. Oskay M, Tamer AU, Azeri C. Antibacterial activity of some actinomycetes isolated from farming soils of Turkey. Afr J Biotechnol. 2004;3(9):441-446.

35. Waksman SA. The Actinomycetes: Classification, Identification and Description of Genera and Species. Baltimore: The Williams and Wilkins Company; 1961. 2:61-292.

36. Bauer AW, Kirby WM, Sherris JC, et al. Antibiotic susceptibility testing by a standardized single disk method. Am J Clin Pathol. 1966;45(4):493496.

37. Hamdali H, Hafidi M, Virolle MJ, et al. Rock phosphate solubilising Actinomycetes: screening for plant growth-promoting activities. World J Microbiol Biotechnol. 2008;24(11):2565-2575.

38. Cappuccino JG, Sherman N. Microbiology: a laboratory manual. 10th ed. India: Pearson education (Singapore); 2004.

39. Gunasekaran P. Laboratory Manual in Microbiology. India: New Age International; 2000.

40. MTCC. Actinomycetes-Lab Manual. Chandigarh: IMTECH; 1998.

41. Shirling EB, Gottlieb D. Methods for characterization of Streptomycetesspecies. Int J Syst Bacteriol. 1966;16:313-340.

42. Bano N, Musarrat J. Characterization of a new Pseudomonas aeruginosa strain $\mathrm{NJ}-15$ as a potential biocontrol agent. Curr Microbiol. 2003;46(5):324-328.

43. You JL, Cao LX, Liu GF, et al. Isolation and characterization of actinomycetes antagonistic to pathogenic Vibrio spp. from nearshore marine sediments. World J Microbiol Biotechnol. 2005;21(5):679-682.

44. Mehta S, Nautiyal CS. An efficient method for qualitative screening of phosphate-solubilizing bacteria. Curr Microbiol. 2001;43(1):51-56.

45. Zhao K, Penttinen P, Guan T, et al. The diversity and anti-microbial activity of Endophytic actinomycetes isolated from medicinal plants in Panxi Plateau, China. Curr Microbiol. 2011;62(1):182-190.

46. Sardi P, Saracchi M, Ouaroni S, et al. Isolation of endophytic Streptomyces from surfacesterilized roots. Appl Environ Microbiol. 1992;58(8):2691-2693.

47. Bascom-Slack CA, Ma C, Moore E, et al. Multiple, novel biologically active endophytic actinomycetes isolated from upper Amazonian rainforests. Microb Ecol. 2009;58(2):374-383.

48. Verma VC, Gond SK, Kumar A, et al. Endophytic actinomycetes from Azadirachta indica A. Juss.: isolation, diversity and anti- microbial activity. Microb Ecol. 2009;57(4):749-756.

49. Taechowisan T, Peberdy JF, Lumyoung S. Isolation of endophytic Actinomycetes from selected plants and their antifungal activity. World $J$ Microbiol Biotechnol. 2003;19(4):381-385.

50. Tsavkelova EA, Klimova SY, Cherdyntseva TA, et al. Microbial producers of plant growth stimulators and their practical use: a review. Appl Biochem Microbiol. 2006;42(2):117-126.

51. Solans M, Vobis G, Cassan F, et al. Production of phytohormones by root associated Saprophytic actinomycetes isolated from the Actinorhizal plant Ochetophila trinervis. World J Microbiol Biotechnol. 2011;27(9):2195-2202.

52. Nimnoi P, Pongsilp N, Lumyong S. Endophytic actinomycetes isolated from Aquilaria crassna Pierre ex Lec and screening of plant growth promoters production. World J Microb Biotechnol. 2010;26(2):193-203.

53. Muller G, Matzanke BF, Raymond KN. Iron transport in Streptomyces pilosus mediated by Ferrichrome siderophores, Rhodotorulic acid, and Enantio-Rhodotorulic acid. J Bacteriol. 1984;160(1):313-318. 
54. Khamna S, Yokota A, Lumyong S. Actinomycetes isolated from medicinal plant rhizosphere soil:Diversity and screening of antifungal compound, indole-3-acetic acid and sidophore production. World $J$ Microbiol Biotechnol. 2009;25:649-655.
55. Tokala RK, Strap JL, Jung CM, et al. Novel plant-microbe rhizosphere interaction involving Streptomyces lydicus WYEC108 and the pea plant (Pisum sativum). Appl Environ Microbiol. 2002;68(5):2161-2171. 